\title{
Observation of Rectifying and Ohmic Grain Boundaries in Polycrystalline BaTiO3 Capacitors with STEM EBIC
}

\author{
William Hubbard, Zachary Lingley, Miles Brodie and Brendan Foran
}

The Aerospace Corporation, El Segundo, California, United States

Transmission electron microscopy (TEM) is the leading technique for studying microelectronic devices at high resolution. TEM primarily provides structural information, related to the type and arrangement of atoms, with spectroscopic attachments, such as energy dispersive x-ray spectroscopy (EDS) and electron energy loss spectroscopy (EELS), providing further details regarding chemistry. Though extremely powerful for many applications, standard TEM capabilities are insufficient for imaging many device features that determine electronic properties but only marginally impact physical and chemical structure.

Electron beam-induced current (EBIC) imaging has been in use since the 1960's [1], usually in a scanning electron microscope (SEM) but occasionally in scanning TEM (STEM). Though EBIC is a general term for current collection as a function of electron beam position, the "standard" mode of EBIC maps current generated by separation of electron-hole pairs (EHPs) by local electric fields. In devices, standard EBIC is used to map local electric fields (e.g. Schottky barriers) and carrier properties [1,2]. A more-recently reported mode of EBIC, called SEEBIC, instead measures holes left in the sample following emission of secondary electrons (SEs) [3]. SEEBIC has been used to map work function [3], resistance, potential, and electric field [4] in devices. Relative to the incident beam, standard EBIC current yield $(>1)$ is generally much larger than SEEBIC yield (a few percent). As a result, SEEBIC has only been reported in STEM, where the high beam energy and thin samples minimize beam absorption and SE production from backscattered electrons [3]. The resolution of SEEBIC (recently demonstrated at $2 \AA$ [5]) is much better than that of standard EBIC due to the small interaction volume associated with SE emission compared to EHP production $[1,2,5]$.

A major barrier to routine characterization of microelectronics with STEM EBIC is sample production; it is difficult to extract a thin sample from a device without compromising its electronic structure, and even more difficult to do so when also electrically connecting to the device. Here we demonstrate STEM EBIC characterization on a sample extracted from multilayer ceramic capacitor (MLCC). A Ni/BaTiO $/ \mathrm{Ni}$ cross section is extracted via standard Ga+ focused ion beam (FIB) techniques and mounted to a TEM biasing chip which contains a silicon nitride membrane with Au electrodes $[4,6]$. The Ni electrodes are each connected, via organometallic ion beam-deposition of $\mathrm{Pt}$, to a pair of $\mathrm{Au}$ electrodes to confirm connectivity to each $\mathrm{Ni}$ electrode. A final 2-kV cleaning is performed to reduce surface damage and contamination.

In the Fig. 1 EBIC image, current is measured on the left Ni electrode, as indicated by the current meter, while the right electrode is held at ground. There are two distinct types of features in the EBIC images: strong local extrema and a weaker contrast that decreases stepwise moving from left to right. The local maxima correlate to standard EBIC current caused by EHP separation from Schottky barriers at some of the grain boundaries (GBs), as shown in the right side of the Fig. 1 diagram. Typical of standard EBIC, the current magnitude is relatively large and its sign depends on the direction of the electric field. A single Schottky barrier produces bright or dark contrast while double Schottky barriers show both contrast polarities flanking the boundary. The weaker, mostly positive (hole) current is indicative of SEEBIC (left side of the Fig. 1 diagram). With the electrode on the right grounded, holes produced during SE emission will reach ground either directly through the right electrode or through the (virtually grounded) current meter via the left electrode. The resulting circuit forms a current divider, and the SEEBIC signal can be recast as a resistance contrast image (RCI) [4, 7], which is superimposed on the standard EBIC signal. In the Fig. 1

STEM EBIC image, the steps in SEEBIC map to resistive GBs, with other boundaries exhibiting relatively low resistance (no SEEBIC steps). 
The Fig. 1 STEM EBIC image shows that not all GBs are highly resistive (some groups of grains show no steps in SEEBIC). EBIC also reveals that not all highly resistive GBs contain Schottky barriers. The evident combination of high- and low-resistance, ohmic and rectifying GBs is at odds with standard reliability models for MLCCs. These models assume that, under bias, all grains contribute equally to the overall dielectric resistance. Exhibiting two distinct EBIC modes, the Fig. 1 STEM EBIC image reveals details about the electronic structure of the capacitor that are not detectable with standard TEM techniques. Unlike standard, two-terminal electrical testing, these spatially-resolved electrical measurements do not average over the large number of grains between Ni layers. Pinpointing these electronic features provides targets for further analysis to determine the physical origins of GB resistivity variation to supplement information gleaned from modeling and statistical analysis.

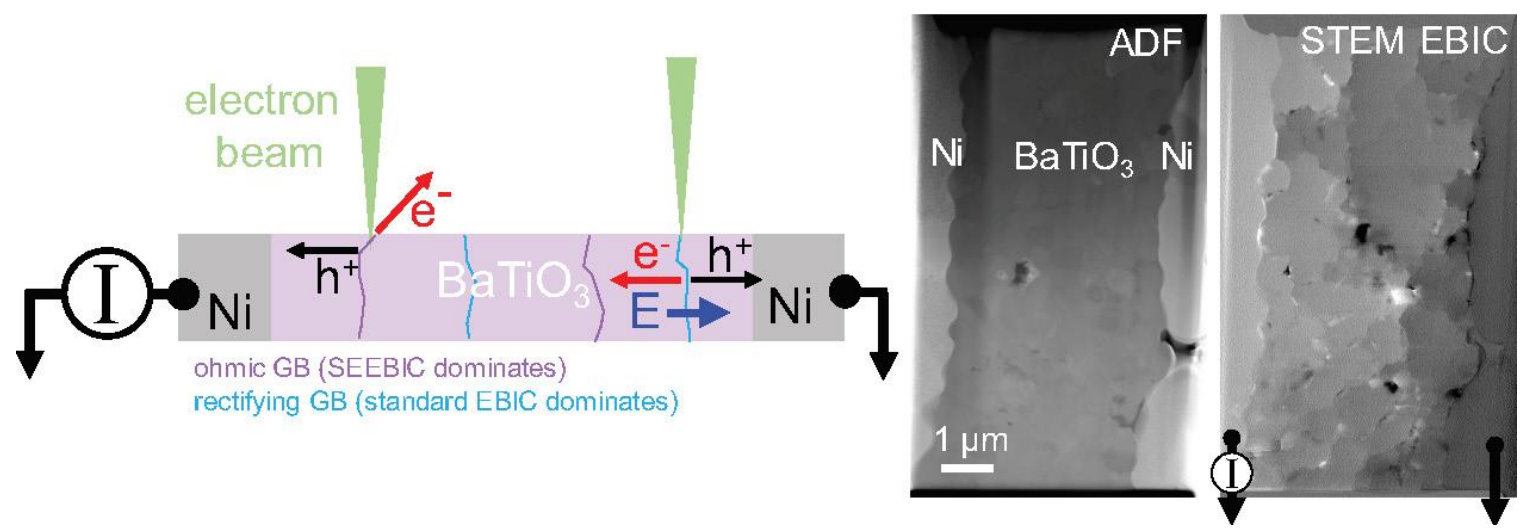

Figure 1. Figure 1. (left) Diagram of EBIC current generation in a Ni/BaTiO3/Ni sample. The left side of the diagram shows generation of SEEBIC on an ohmic (purple) GB. The right side of the diagram shows standard EBIC acquired on a rectifying (blue) GB, which contains a Schottky barrier. Both EBIC modes may be present in a single GB. (right) Annular dark field (ADF) STEM and STEM EBIC images of Ni/BaTiO3/Ni capacitor cross section. EBIC is measured on the left $\mathrm{Ni}$ electrode and the right electrode is grounded. The stepwise decrease in EBIC, moving left to right, corresponds to steps in resistance as detected with SEEBIC. The generally brighter local extrema are indicative of Schottky barriers detected with standard EBIC.

\section{References}

[1] TE Everhart, OC Wells, and RK Matta, Proceedings of the IEEE, 52 (1964). p. 1642-1647

[2] HJ Leamy, Journal of Applied Physics, 53 (1982), p. R51-R80.

[3] WA Hubbard et al, Physical Review Applied, 10 (2018), p. 044066.

[4] WA Hubbard et al, Applied Physics Letters, 115 (2019), p. 133502.

[5] M Mecklenburg et al, Ultramicroscopy, 207 (2019), p. 112852.

[6] M Mecklenburg et al, Microscopy and Microanalysis, 19 (2013), p. 458-459.

[7] CA Smith et al, IEEE Transactions on Electron Devices, 33 (1986), p. 282-285. 